\title{
DERIVED STRENGTHS OF PREFERENCE RELATIONS ON COORDINATES *
}

\author{
Peter WAKKER \\ University of Nijmegen, $6500 \mathrm{HE}$ Nijmegen, The Netherlands
}

Received 20 May 1988

Accepted 12 July 1988

\begin{abstract}
A way is indicated to derive, from a preference relation on a Cartesian product, strength of preference relations on the coordinate sets. These strengths of preference relations are then used to reformulate several well-known properties of preference relations, and make their meaning more transparent. A new result for dynamic contexts is given.
\end{abstract}

\section{Introduction}

This paper examines the existence of several kinds of additively decomposable representing functions for preference relations on Cartesian products. This is done under the assumption that the coordinate sets are connected topological spaces, e.g., $\mathbb{R}_{+}^{m}$, or $\mathbb{R}$. The representing functions which we shall find will be cardinal.

Several authors have dealt with cardinal additively decomposable representing functions under the assumption that the coordinate sets are 'mixture spaces', the main intended examples of mixture spaces being sets of probability distributions. See, for instance, Anscombe and Aumann (1963), Fishburn (1965), Pollak (1967), Keeney and Raiffa (1976, Karni, Schmeidler and Vind (1983). Usually the axioms of von Neumann and Morgenstern (1953, ch. 3 and the appendix) are assumed, leading to linear representing functions. Cardinality is easily obtained in the presence of linearity because, if one linear function is a (strictly increasing) transformation of another linear function, then in fact it must be a linear transformation. Hence, if two linear functions induce the same preference relation, then they also induce the same strength of preference relation.

Matters are more complicated if the assumption of linearity is weakened to the assumption of continuity. To see this, let $f$ and $g$ be two continuous functions on a connected domain. Let $f=\varphi \cdot g$ for a transformation $\varphi$. For guaranteeing that $\varphi$ is (positively) linear, the assumption of strict increasingness of $\varphi$ no longer suffices. It must be strengthened to the more complicated assumption that $\varphi$ preserves ('first-order') differences, as shown in Basu (1982). The characterizing properties for preference relations on connected topological spaces, met in literature, reflect this additional complication.

This paper handles the additional complication by indicating a way to derive, from a preference relation on a Cartesian product ('revealed'), strength of preference relations on the coordinate sets. By means of these we formulate several properties and results, met in literature, for connected topological spaces. All reformulated versions will require that certain strengths of preferences,

\footnotetext{
* This paper was prepared while visiting the Department of Economics of the Tel-Aviv University.
} 
derived from the preference relation, do not reveal contradictions. We think the reformulated versions are more appealing, in clarifying the first-order difference idea which is always underlying continuous cardinal utility.

Also we use the strength of preference relations to obtain a new result for dynamic contexts. Koopmans (1972) gave a characterization of additively decomposable representing functions with constant discount factors, mainly by means of a stationarity assumption. This requires an infinite set of (coordinates) points of time, $\mathbb{N}$ in Koopmans' paper. Grodal (1978, Theorem 4) has given a result for continuous time. We obtain a result for individuals who deal with only a finite number of points of time, as considered for instance in Samuelson (1958). Zilcha (1988) is a recent survey, indicating the usefulness in intergenerational models of finiteness of the number of points of time for each individual.

\section{Preferences and strengths of preferences}

This section gives elementary definitions and notations. Further it indicates the way to derive the strength of preference relations from the preference relation.

Let $n \in \mathbb{N}$. Let $\mathscr{C}_{1}, \ldots, \mathscr{C}_{n}$ be connected topological spaces. The set of alternatives is the Cartesian product $X_{i=1}^{n} \mathscr{C}_{i}$. It is endowed with the product topology, which is connected too.

Let $\geqslant$ be a binary relation on $X$, the preference relation of a decision maker. We write $x \leqslant y$ if $y \geqslant x$. As usual $>=\geqslant \backslash \preccurlyeq$, and $\approx=\succcurlyeq \cap \preccurlyeq$. We write $x \prec y$ if $y \succ x$. Further, $\succcurlyeq$ is continuous if $(x: x \geqslant y)$ and $(x: x \leqslant y)$ are closed sets for all $y \in X$. A weak order $\geqslant$ is complete $(x \geqslant y$ or $y \geqslant x$ for all $x, y \in X)$ and transitive. A function $V: X \rightarrow \mathbb{R}$ represents $\geqslant$ if $[x \geqslant y \Leftrightarrow V(x) \geq V(y)]$ for all $x, y \in X . V: X \rightarrow \mathbb{R}$ is additively decomposable if there exist $V_{j}: \mathscr{C}_{j} \rightarrow \mathbb{R}, j=1, \ldots, n$, such that $V: x \mapsto \sum_{j=1}^{n} V_{j}\left(x_{j}\right)$. Here, as usual, $x_{j}$ is the $j$ th coordinate of $x$.

We write $x_{-i} v_{i}$ for $x$ (with $x_{i}$ replaced by $v_{i}$ ), and for $i \neq j,\left(x_{-i, j} v_{i}, w_{j}\right.$ ) for $x$ (with $x_{i}$ replaced by $v_{i}$, and $x_{j}$ by $\left.w_{j}\right)$.

Coordinate (or index) $i$ is non-essential if $x_{-i} v_{i} \approx x$ for all $x, v_{i}$; otherwise $i$ is essential.

The following definitions give the main tools in this paper.

Definition 1. The quaternary relation $\succ_{i}^{* *}$ on $\mathscr{C}_{i}$, with $x_{i} y_{i} \succ_{i}^{* *} v_{i} w_{i}$ meaning $\left(x_{i}, y_{i} v_{i} w_{i}\right) \in \succ_{i}^{* *}$, is defined by

$$
\begin{aligned}
x_{i} y_{i} \succ_{i}^{* *} v_{i} w_{i} \text { if } a_{-i} x_{i} \succcurlyeq b_{-i} y_{i} \text { and } \\
\qquad a_{-i} v_{i} \prec b_{-i} w_{i} \text { for some alternatives } a, b .
\end{aligned}
$$

For an intuitive elucidation, suppose for simplicity that $x_{i}$ is better than $y_{i}$, and $v_{i}$ is better than $w_{i}$. Then $a_{-i} v_{i} \prec b_{-i} w_{i}$ suggests that the positive argument, to obtain $v_{i}$ instead of $w_{i}$ on coordinate $i$, does not outweigh the obviously negative argument for the left alternative against the right alternative, as yielded by the coordinates $j \neq i$. And $a_{-i} x_{i} \geqslant b_{-i} y_{i}$ suggests that the positive argument, to obtain $x_{i}$ instead of $y_{i}$, does outweigh the same (negative) argument. We interpret this to mean that it has been revealed from $\geqslant$, that the strength of preference of $x_{i}$ over $y_{i}$ exceeds that of $v_{i}$ over $w_{i}$.

A further definition: 
Definition 2. The quaternary relation $\geqslant_{i}^{*}$ on $\mathscr{C}_{i}$, with $x_{i} y_{i} \geqslant_{i}^{*} v_{i} w_{i}$ meaning $\left(x_{i}, y_{i}, v_{i}, w_{i}\right) \succcurlyeq_{i}^{*}$, is defined by

$$
\begin{aligned}
x_{i} y_{i} \succcurlyeq_{i}^{*} v_{i} w_{i} \text { if } a_{-i} x_{i} \succcurlyeq b_{-i} y_{i} \text { and } \\
a_{-i} v_{i} \preccurlyeq b_{-i} w_{i} \text { for some alternatives } a, b .
\end{aligned}
$$

The introduced binary relations in general do not have to satisfy properties such as completeness or transitivity. And $>_{i}^{* *}$ in no way has to be the asymmetric part of $\geqslant_{i}^{*}$; see Theorem 3.1.

\section{Characterizations through strength of preferences}

Throughout this section we assume that $\geqslant$ is a continuous weak order on $X$. We shall list several properties of $\succcurlyeq$, give their equivalent formulations in terms of $\succcurlyeq_{i}^{*}$ and $\succ_{i}^{* *}$, and give the main theorems in which the properties are used.

We say that $\geqslant$ satisfies triple cancellation if

$$
\left[\begin{array}{l}
\text { For all } i, a, \ldots, w_{i} \\
a_{-i} x_{i} \preccurlyeq b_{-i} y_{i} \text { and } \\
a_{-i} v_{i} \geqslant b_{-i} w_{i} \text { and } \\
c_{-i} x_{i} \geqslant d_{-i} y_{i} \text { imply } \\
c_{-i} v_{i} \geqslant d_{-i} w_{i}
\end{array}\right] \text { which is iff }\left[\begin{array}{l}
\text { For all } i, x_{i}, \ldots, w_{i}, \\
v_{i} w_{i} \geqslant_{i}^{*} x_{i} y_{i}, \\
\text { then not } \\
x_{i} y_{i} \succ_{i}^{* *} v_{i} w_{i}
\end{array}\right] \text {. }
$$

Theorem 1. Let at least two coordinates be essential. There exists a continuous additively decomposable representing function $V$ for $\geqslant$, if and only if $\geqslant$ satisfies triple cancellation. $V$ is cardinal.

Proof. For the case of exactly two essential coordinates this can be derived from Chapter 6 of Krantz, Luce, Suppes and Tversky (1971), as indicated in Wakker (1986b, Theorem 4.4). For the case of three or more coordinates this follows straightforwardly from Theorem 2 below $\left(a_{-i} x_{i} \leqslant a_{-i} x_{i}\right.$ and $a_{-i} y_{i} \succcurlyeq a_{-i} y_{i}$ give $x_{i} x_{i} \preccurlyeq_{i}^{*} y_{i} y_{i}$; (1) forbids $x_{i} x_{i} \succ_{i}^{* *} y_{i} y_{i}$ ).

Analogues of the above theorem (and of the one below) for the case where coordinate sets are sets of probability distributions, are given in Fishburn (1965), Pollak (1967), and Keeney and Raiffa (1976, Theorem 6.4).

We say that $\geqslant$ satisfies the sure-thing principle (other terms are strong separability, preferential/ coordinate independence) if

$\left[\begin{array}{l}\text { For all } i, a, b, x_{i}, y_{i} \\ a_{-i} x_{i} \geqslant b_{-i} x_{i} \text { implies } \\ a_{-i} y_{i} \geqslant b_{-i} y_{i}\end{array}\right]$ which is iff $\left[\begin{array}{l}\text { For no } i, x_{i}, y_{i}, \\ x_{i} x_{i} \succ_{i}^{* *} y_{i} y_{i}\end{array}\right]$.

Theorem 2. Let at least three coordinates be essential. There exists a continuous additively decomposable representing function $V$ for $\succcurlyeq$, if and only if $\geqslant$ satisfies the sure-thing principle. This $V$ is cardinal.

Proof. See Theorem 4.1 in Wakker (1986b), which generalizes Theorem 3 of Debreu (1960) by dropping the assumption of topological separability, and Theorem 6.14 of Krantz, Luce, Suppes and Tversky (1971) by also deriving continuity. 
For the next property and theorem it is assumed that $\mathscr{C}_{1}=\mathscr{C}_{2}=, \ldots,=\mathscr{C}_{n}=\mathscr{C}$ for some connected topological space $\mathscr{C}$. The present formulation by means of strength of preference has been indicated in section 3 of Wakker (1984a).

We say that $\geqslant$ satisfies cardinal coordinate independence $(\mathrm{CCI})$ if

$\left[\begin{array}{l}\text { For all } j, \text { essential } i \\ a, b, c, d \in X, \text { and } \\ a, \beta, \gamma, \delta \in \mathscr{C}, \\ a_{-i} \alpha \preccurlyeq b_{-i} \beta \text { and } \\ a_{-i} \gamma \geqslant b_{-i} \delta \text { and } \\ c_{-j} \alpha \geqslant d_{-j} \beta \text { imply } \\ c_{-j} \gamma \geqslant d_{-j} \delta\end{array}\right]$ which is iff $\left[\begin{array}{l}\text { For all } j, \text { essential } i, \\ \alpha, \beta, \gamma, \delta, \in \mathscr{C}: \\ \gamma \delta \succcurlyeq_{i}^{*} \alpha \beta \\ \text { then not } \\ \alpha \beta \succ_{j}^{* *} \gamma \delta\end{array}\right]$.

Note the difference between (3) and (1), apart from the unimportant detail of essentially of $i$. Formula (3) requires strength of preferences to be 'non-contradictory' across, possibly, different coordinates.

Theorem 3. Let $\mathscr{C}_{i}=\mathscr{C}$ for all $i$, and let at least two coordinates be essential. Then there exist non-negative $p_{1}, \ldots, p_{n}$, summing to one, and a continuous $U: \mathscr{C} \rightarrow \mathbb{R}$, such that $x \mapsto \sum p_{j} U\left(x_{j}\right)$ represents $\geqslant$, if and only if $\succcurlyeq$ satisfies cardinal coordinate independence. $U$ is cardinal, the $p_{1}, \ldots, p_{n}$ are unique.

Proof. See Wakker (1984a, Theorem 5.1).

When applied to the context of decision making under uncertainty, the above theorem gives a characterization of subjective expected utility maximization. It differs from Savage (1954) in dealing with a finite state space. Its analogue for the case where $\mathscr{C}$ is a set of probability distributions as given in Anscombe and Aumann (1963). Anscombe and Aumann do not consider a Cartesian product of marginal distributions, but rather consider simultaneous distributions. Their Assumption 2 allows one to consider only the marginal distributions.

For the next property and theorem we shall assume that $\mathscr{C}_{i}=\mathbb{R}$ for every $i$. The following property reflects the idea of non-increasing marginal utility.

We say that $\geqslant$ satisfies the concavity assumption if

$$
\left[\begin{array}{l}
\text { For all } i, x, y, \\
\text { and real } \alpha, \beta, \epsilon \\
\text { with either } \alpha \geq \beta \\
\text { and } \epsilon \geq 0, \\
\text { or } \alpha \leq \beta \text { and } \epsilon \leq 0, \\
x_{-i} \alpha \geqslant y_{-i} \beta \text { implies } \\
x_{-i}(\alpha-\epsilon) \geqslant y_{-i}(\beta-\epsilon)
\end{array}\right] \text { which is iff }\left[\begin{array}{l}
\text { For all } i \\
\text { and real } \alpha, \beta, \epsilon, \\
\text { with either } \alpha \geq \beta \\
\text { and } \epsilon \geq 0 \\
\text { or } \alpha \leq \beta \text { and } \epsilon \leq 0, \\
\text { not } \alpha \beta \succ_{i}^{* *}(\alpha-\epsilon)(\beta-\epsilon)
\end{array}\right]
$$

Theorem 4. Let $\mathscr{C}_{i}=\mathbb{R}$ for all $i$, and let at least three coordinates he essential. Then there exists a concave additively decomposable representing function $V$ for $\succcurlyeq$, if and only if $\succcurlyeq$ satisfies the concavity assumption. This $V$ is cardinal. 


\section{Proof. See Wakker (1986).}

It is straightforwardly shown that in the above theorem 'concave' can be replaced by 'strictly concave', if in (4) for every $\epsilon \neq 0$ we have: not $\alpha \beta \succcurlyeq_{i}^{*}(\alpha-\epsilon)(\beta-\epsilon)$. Yaari (1978) studied versions of (4) for $\alpha \neq \beta$ and $\epsilon \neq 0$.

In the following property and theorem, it is assumed, slightly more generally than Koopmans (1972), that $\mathscr{C}_{1}=\ldots=\mathscr{C}_{n}=\mathscr{C}$, for a connected topological space $\mathscr{C}$; e.g., $\mathscr{C}=\mathbb{R}$, standing for money. Further we use the term 'point of time' for index. The property below is a reformulation of its version in Wakker (1984b).

We say that point of time $j$ is CCI-related to point of time $i$ if, for all $\alpha, \beta, \gamma, \delta$

$\alpha \beta \succcurlyeq_{i}^{*} \gamma \delta \Rightarrow \operatorname{not} \gamma \delta \succ_{j}^{* *} \alpha \beta$.

Theorem 5. Let $n \geq 3$. Let all points of time $1, \ldots, n$ be essential. Then the following two statements are equivalent for the continuous weak order $\geqslant$ on $\mathscr{C}^{n}$ :

(i) There exists a continuous $U: \mathscr{C} \rightarrow \mathbb{R}$, and a $\lambda$ in $(0,1]$, such that $x \mapsto \sum_{j-1}^{n} \lambda^{j} U\left(x_{j}\right)$ represents $\geqslant$.

(ii) Every point of time $j \geq 2$ is CCI-related to its predecessor $j-1$; there exist $(\alpha, \ldots, \alpha) \succcurlyeq(\beta, \ldots, \beta)$ $>(\theta, \ldots, \theta)$ such that, for every point of time $j<n,\left[(\theta, \ldots, \theta)_{-j} \beta\right]=\left[(\theta, \ldots, \theta)_{-(j+1)} \alpha\right]$; and $\gamma \succ_{1}^{* *} \delta \delta$ for no $\gamma, \delta \in \mathscr{C}$.

Furthermore, the ('discount factor') $\lambda$ in (i) is uniquely determined; $U$ is cardinal.

Sketch of the proof. Since (i) $\Rightarrow$ (ii) is straightforward, we assume (ii), and derive (i) and the 'Furthermore'-statement. For every $j \geq 2$, and any $x, \gamma, \delta, x_{-(j-1)} \delta t_{-(j-1)} \delta$ and $x_{-(j-1)} \gamma \preccurlyeq$ $x_{-(j-1)} \gamma$, hence by (5) $\gamma \succ_{j}^{* *} \delta \delta$ for no $\gamma, \delta \in \mathscr{C}$. We may apply Theorem 2 , to obtain a representing function $V$ : $x \mapsto \Sigma V_{j}\left(x_{j}\right)$. We may set $V_{j}(\theta)=0$ for all $j$.

Since every $j$ is CCI-related to $j-1$, there can be seen to exist $\lambda_{j}$ such that $V_{j}=\lambda_{j} V_{j-1}$ for all $j$. This is much like in the proof of Theorem 2.1 in Wakker (1984a). In short, the idea is that, for every $j \geq 2$, and $\eta \in \mathscr{C}$, there exists an open neighbourhood of $\eta$ on which $V_{j}$ orders differences as $V_{j-1}$ does it. Hence locally $V_{j}$ can be seen to be a positive affine transformation of $V_{j-1}$. Then it must also be globally. Now $V_{j}(\theta)=0=V_{j-1}(\theta)$ implies the existence of $\lambda_{j}$ as above.

Substitution of the preferences in (ii) concerning $\alpha, \beta$, and $\theta$, shows that, with $\lambda$ : $=\lambda_{2}$, we have $\lambda_{j}-\lambda$ for all $j$; and that $0 \leq \lambda \leq 1$. Since all points of time are essential, $\lambda-0$ cannot be. Finally, set $U:=V_{1} / \lambda$.

The furthermore-statement follows from the cardinality of $V$ in Theorem 2.

For an interpretation of (ii) above, say consequences are amounts of money, $\mathscr{C}=\mathbb{R}$. The CCI-relatedness condition says that revealed strengths of preferences concerning money on a point of time, should not contradict revealed strength of preferences on the previous point of time. For the condition concerning $\alpha, \beta$, and $\theta$, say $\theta$ is the status quo, receive $\$ 0$. Let $\alpha$ be come standardized amount of money, say $\$ 1$. The condition says that today's equivalent $\$ \beta$, for tomorrow's receipt of $\$ 1$, should be the same on every today. This condition weakens Koopman's stationary assumption.

One can replace $(0,1]$ in (i) above by $(0,1)$, if in (ii) one replaces $(\alpha, \ldots, \alpha) \succcurlyeq(\beta, \ldots, \beta)$ by $(\alpha, \ldots, \alpha) \succ(\beta, \ldots, \beta)$.

\section{Conclusion}

It is desirable to formulate results, as much as possible, in terms of primitives of an adopted model. In decision theory the primitives are the set of alternatives, structure on this set, and the 
preference relation of a decision maker. Derived notions (such as utility functions) should be adopted in the formulation of results, only if this achieves a significant intuitive gain. We hope that the derived strength of preference relations, as defined in this paper, do give a significant intuitive gain in the derivation of continuous cardinal utility.

\section{References}

Anscombe, F.J. and R.J. Aumann, 1963, A definition of subjective probability, Annals of Mathematical Statistics 34, $199-205$. Basu, K., 1982, Determinateness of the utility function: revisiting a controversy of the thirties, Review of Economic Studies 49 . 307-311.

Debreu, G., 1960, Topological methods in cardinal utility theory, in: K.J. Arrow, S. Karlin and P. Suppes, eds., Mathematical methods in the social sciences (Stanford University Press, Stanford, CA) 16-26.

Fishburn, P.C., 1965, Independence in utility theory with whole product sets, Operations Research 13, 28-45.

Grodal, B., 1978, Some further results on integral representation of utility functions (Institute of Economics, University of Copenhagen, Copenhagen). Forthcoming in: K. Vind, Independent preferences.

Karni, E., D. Schmeidler and K. Vind, 1983, On state dependence preferences and subjective probabilities, Econometrica 51, 1021-1031.

Keeney, R.L. and H. Raiffa, 1976, Decisions with multiple objectives (Wiley, New York).

Koopmans, T.C., 1972, Representations of preference orderings with independent components of consumption; (and:) Representations of preference orderings over time, in: C.B. McGuire and R. Radner, eds., Decision and organization (North-Holland, Amsterdam) 57-100.

Krantz, D.H., R.D. Luce, P. Suppes and A. Tversky, 1971, Foundations of measurement, Additive and polynomial representations, Vol. I (Academic Press, New York).

Pollak, R.A., 1967, Additive von Neumann-Morgenstern utility functions, Econometrica 35, 485-494.

Samuelson, P.A., 1958, An exact consumption-loan model of interest with or without the social contrivance of money, The Journal of Political Economy 46, 467-482.

Savage, L.J., 1954, The foundations of statistics (Wiley, New York), Second ed., 1972 (Dover, New York).

von Neumann, J. and O. Morgenstern, 1944, 1947, 1953, Theory of games and economic behavior (Princeton University Press, Princeton, NJ).

Wakker, P.P., 1984a, Cardinal coordinate independence for expected utility, Journal of Mathematical Psychology 28, $110-117$.

Wakker, P.P., 1984b, Continuous expected utility for arbitrary state spaces, Methods of Operations Research, 50, 113-129.

Wakker, P.P., 1986, Concave additively decomposable representing functions and risk aversion, in: L. Daboni, A. Montesano and M. Lines, eds., Recent developments in the foundations of utility and risk theory (Reidel, Dordrecht) $249-262$.

Zilcha, I, 1988, Intergenerational transfers, in: J. Eatwell and P. Newman, eds., The new Palgrave: A dictionary of economic theory and doctrine (Macmillan Press, Cambridge) forthcoming. 